$R M x A C, \mathbf{5 2}, 27-27(2020)$

(C) 2020: Instituto de Astronomía, Universidad Nacional Autónoma de México

https://doi.org/10.22201/ia.14052059p.2020.52.11

\title{
ORBIT DETERMINATION BASED ON SUN SENSOR AND MAGNETOMETER FOR NANOSATELLITE APPLICATIONS
}

\author{
L. R. Colpari Carrizo ${ }^{1}$
}

This work focuses on an orbit determination algorithm design based on low cost sensors for small size satellites.

Nanosatellite platforms are becoming more important as there is a growing trend to miniaturise satellites. Due to the small size of these platforms, there are several constraints in the design of these satellites. One key requirement to achieve mission success is a robust and reliable orbit determination. This work focuses on an orbit determination algorithm design based on low cost components that have lower accuracy and higher noise than space qualified components. The advantages of the sensor based orbit determination are that many of the required sensors may already be on the satellite, requiring little additional space (Pereyra et al. 2017; Psiaki 1998). The sensor measurements are taken and processed by an estimator and propagator known as the Extended Kalman Filter (EKF).

Implementation: The main force governing the motion of a satellite orbiting Earth is our planet's gravitational field. The perturbation due to Earth's non-symmetric mass distribution can be sufficiently modelled with the J2 effect only, since at an altitude around $700 \mathrm{~km}$. it is at least 400 times larger than other ones. All representations of the satellite dynamics will be approximations to some extent. The assumption lies in that the sources of error of that approximation cancel each other out over time. The system and measurement equations are now functions depending on the current state, control input and noise: The EKF works by linearizing the system about the current state estimate. The math and statistics are discussed in the references, uncertainties can be summarized as being of zero-mean Gaussian. The measurements have to be manipulated and turned into model-measurements, the first one is the absolute value of the measured magnetic field $B_{\text {meas }}$, the second one is the measured cosine of the angle between the magnetic field and the direction towards the sun $S_{\text {meas }}$. The measurement readings can be summarized as a function of the current state and the random noise variables $y$, where

\footnotetext{
${ }^{1}$ Universidad Autónoma Juan Misael Saracho, Tarija, Bolivia (colparic.ricardo@uajms.edu.bo).
}

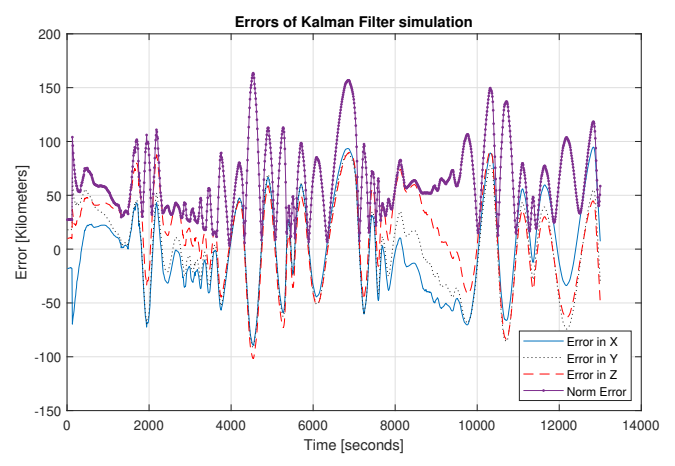

Fig. 1. For a magnetometer accuracy of $500 \mathrm{nT}$, Sun sensor error of $0.2 \mathrm{deg}$, step size of $1 \mathrm{~km}$, and $10 \mathrm{~s}$ sampling, the filter converges to an RMS error about $68 \mathrm{~km}$.

the vector $v_{y}$ is a combination of the noises of both model-measurements.

$$
\begin{aligned}
& y_{1}=\sqrt{B_{\text {meas }}^{T} B_{\text {meas }}} \approx \sqrt{B_{\text {model }} B_{\text {model }}}+v_{y 1} \\
& y_{2}=\frac{B_{\text {meas }}^{T} S_{\text {meas }}}{\sqrt{B_{\text {meas }}^{T} B_{\text {meas }}}} \approx \frac{B_{\text {model }}^{T} S_{\text {model }}}{\sqrt{B_{\text {model }} B_{\text {model }}}}+v_{y 2}
\end{aligned}
$$

Simulations: The EKF is tested using the CelestLab library of space mechanics functions written in the Scilab environment, the "true" position and sensor measurements are obtained with the $\mathrm{Xcos}$ Aerospace Block set with a Lyddane analytic propagator, same with the sun direction and magnetic field simulation, the values of the true position are obtained as well as simulated measurements from the sensors, that are necessary to initialize and run the algorithm which results can be seen in the Figure 1.

Conclusions: The present work implements an orbit determination based on the measurements of the magnetic field and sun direction vector for nanosatellite sensors, this is advantageous as a backup in the event of failure of the primary orbit determination system, whether a failure of GNSS receiver or the inability to upload replacement TLEs.

\section{REFERENCES}

Pereyra, M., Alonso, R., \& Kuba, J. 2017, in Development of Sensor orbit based determination LASSS, p. 10

Psiaki, M. L. 1998, in Autonomous LEO Orbit Determination From Magnetometer and Sun Sensor Data 